\title{
Voltage Spike Detection in High Field Superconducting Accelerator Magnets
}

\author{
D.F. Orris, R. Carcagno, S. Feher, A. Makulski, Y.M. Pischalnikov
}

\begin{abstract}
A measurement system for the detection of small magnetic flux changes in superconducting magnets, which are due to either mechanical motion of the conductor or flux jump, has been developed at Fermilab. These flux changes are detected as small amplitude, short duration voltage spikes, which are $\sim 15 \mathrm{mV}$ in magnitude and lasts for $\sim 30 \mu s e c$. The detection system combines an analog circuit for the signal conditioning of two coil segments and a fast data acquisition system for digitizing the results, performing threshold detection, and storing the resultant data. The design of the spike detection system along with the modeling results and noise analysis will be presented. Data from tests of high field Nb3Sn magnets at currents up to $\sim 20 \mathrm{KA}$ will also be shown
\end{abstract}

Index Terms-Superconducting, Magnet, Flux Jump, spike, High Field

\section{INTRODUCTION}

$\mathrm{T}$ here have been many observations of voltage spikes produced from either mechanical motion of the superconductor or from flux jump. However, it has been challenging to confirm and systematically study such events due to both the limitations of the data acquisition system used to capture the events as well as the poor signal-to-noise ratio (SNR) of the spike voltage signals produced by the magnet under test. Early measurements at Fermilab made use of a secondary trigger capability of a Digital Quench Detection (DQD) system [1], whose digitally filtered data allowed lower threshold arming for the exclusive purpose of recording such events. Some of those events were confirmed by the measurement of coincidental disturbances in the magnetic field via a multipole harmonic measurement probe. Such voltage spike information became useful feedback for the development of prototype superconducting accelerator magnets. In addition, LBNL and CERN have also been successful at detecting such spikes in their superconducting magnets [2].

The study of transient voltage spikes produced by high field magnets being developed at Fermilab is of interest, but due to the spike's small magnitude and relatively high frequency they have proven very challenging to detect. This is also due in part

Manuscript received October 4, 2004. This work was supported by the U.S. Department of Energy.

Authors are with the Fermi National Accelerator Laboratory, Batavia, IL 60510 USA. to the power supply ripple and ringing caused by the power supply SCR switching [3], which is approximately the same magnitude and occurs in the same frequency range as the voltage transients of interest. Also, the isolation amplifiers used in the existing DAQ system were band limited to $<5 \mathrm{KHz}$. As a result, the existing DQD system was unable to confidently detect these spike signals. For this reason and because of its potential use as a tool for providing important engineering feedback for magnet development, a new voltage spike detection system has been developed at Fermilab.

\section{Design of the New Voltage Transient (SPIKe) DETECTION SYSTEM}

\section{A. General System Requirements}

The signal of interest is normally produced in one of the superconducting magnet half coils. There are two mechanisms thought to generate these signals: 1) mechanical motion of the conductor; and 2) flux jump. Common mode signals generated outside the coil will produce inductive voltages of the same polarity across each half coil, but transient voltages generated by the conductor will have the opposite polarity in the half coils. This is important since bucking of the two half coil signals will reject common mode noise signals leaving the transient signal of interest intact. For this reason it is equally important that the two channels of the spike detection system have a matched phase and amplitude response in order to maximize common mode rejection throughout the frequency range of interest.

The detection system must be able to detect transient voltages on the order of $15 \mathrm{mV}$ in the frequency range of $1.5 \mathrm{KHz}$ to $30 \mathrm{KHz}$ between two superconducting half coils whose inductance is from $\sim 15 \mu \mathrm{H}$ to $1 \mathrm{mH}$. Since the transient voltages can be produced at any current, the detection system must be able to measure these signals throughout the power supply range: 0 to $30 \mathrm{KA}$ at 0 to $30 \mathrm{~V}$ and be protected up to $1 \mathrm{kV}$ to ground when the magnet energy extraction resistor is enabled.

In order to prevent ground loops and protect against high common mode voltages the data acquisition system must be isolated from the power system bus. In addition, the frequency response of the isolation amplifiers must be matched in order to satisfy the bucking requirements.

The specification of the magnet voltage tap wires is also important in order to prevent both inductive and capacitive 
coupling with other voltage taps or the magnet. Such noise voltage is generally uncorrelated and would not be easily rejected. Therefore, the careful routing of the wires, twisting, and shielding are all specified for each magnet to be tested. The cabling of the detection system is also specified carefully to prevent ground loops, etc.

Since the half coil signals are digitally bucked the performance of the data acquisition system's analog-to-digital converters (ADC) must have excellent gain and stability characteristics, and must sample at least twice the frequency of interest in order to satisfy the Nyquist criterion.

\section{B. Design Description}

To satisfy these requirements a spike detection system was developed using high quality components. The front-end electronics was designed and assembled in-house and the data acquisition (DAQ) system was purchased commercially. The software for the DAQ was written in house. A block diagram of the spike detection circuit is shown in Fig. 1. Special attention was taken in selecting the passive components that determines the frequency response of both channels. This was done to assure that the two coil channels have identical temperature and time stable frequency characteristics.

The input signal is first conditioned by a low pass filter,

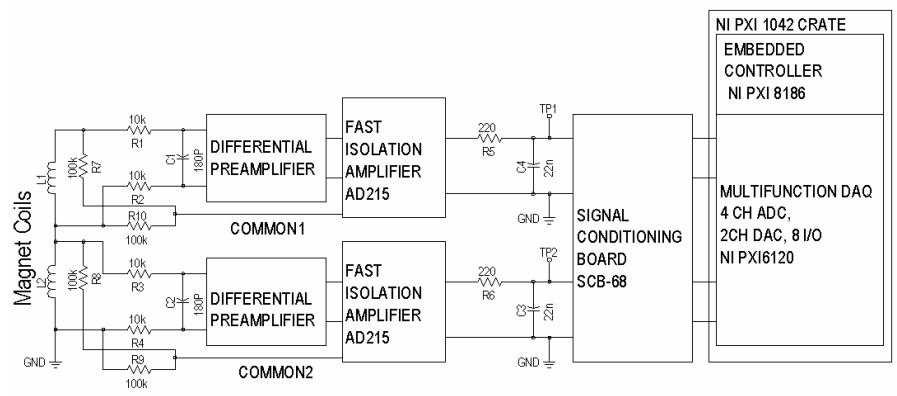

Fig. 1. Voltage transient "Spike" detection circuit block diagram.

which attenuates frequencies greater than $30 \mathrm{KHz}$ and also blocks RF noise to the preamplifier preventing harmonic distortion and rectification of the signal.

The input differential pre-amplifiers provide the major amplification of the signal. The gain is jumper selectable and the range of the gain is from 5 to 100 . These amplifiers were placed upstream of the fast isolation amplifiers in order to increase the signal magnitude before isolation, therefore improving the signal to noise ratio (SNR).

The isolation amplifier component chosen for this circuit is the Analog Devices AD215. It has a $1500 \mathrm{~V}$ isolation voltage rating, a wide bandwidth of $120 \mathrm{KHz}$, and a low harmonic distortion of $-80 \mathrm{~dB}$. A second filter at the output of the isolation amplifiers filters out high frequency modulation noise produced by the isolation amplifiers - typically $\sim 2.5 \mathrm{mVpp}$ (peak-to-peak voltage). Together with the input filter, this forms a $2^{\text {nd }}$ order low pass filter for the input signal.

The signal-conditioning board (SCB) is commercially available from National Instruments, SCB-68, and its output is digitized by the National Instruments PXI multifunction DAQ: NI PXI-6120. This module has 4 channels of simultaneous
Analog to Digital Converters (ADC), 2 channels of Digital to Analog Converters (DAC), 8 channels of input/output (I/O), and 2 counter/timers. The ADC has 16-bit resolution, built-in anti-aliasing filters, and can sample up to $800 \mathrm{KHz}$.

The control software for this system is written in Labview, and runs under the National Instruments real-time environment of the PXI controller. The main purpose of this software is to perform filtering, digital bucking, and triggering for data storage along with pseudo real-time visualization of the data with FFT's of the current and bucked magnet halfcoils signal.

\section{Modeling of the Magnet Under Test}

The first measurements of the completed system using a superconducting dipole magnet showed that the bucking of the half coils was not good enough to detect a typical transient spike. This was especially true for higher inductance magnets. In order to determine the cause of this behavior, it was decided to characterize the magnitude of the bucking signal as a function of frequency. A test was performed using a constant amplitude AC voltage source to excite the magnet. For this test a peek-to-peek sinusoidal excitation voltage of $3 \mathrm{~V}$ was generated at many frequencies to cover the entire bandwidth of the spike detection system. The voltage of the two coil halves was digitized along with the bucked signal to determine the magnitude response. Fig. 2 represents the schematic used for this simulation. This schematic also includes blocks that represent signal cables and the input filters to the isolation amplifiers.

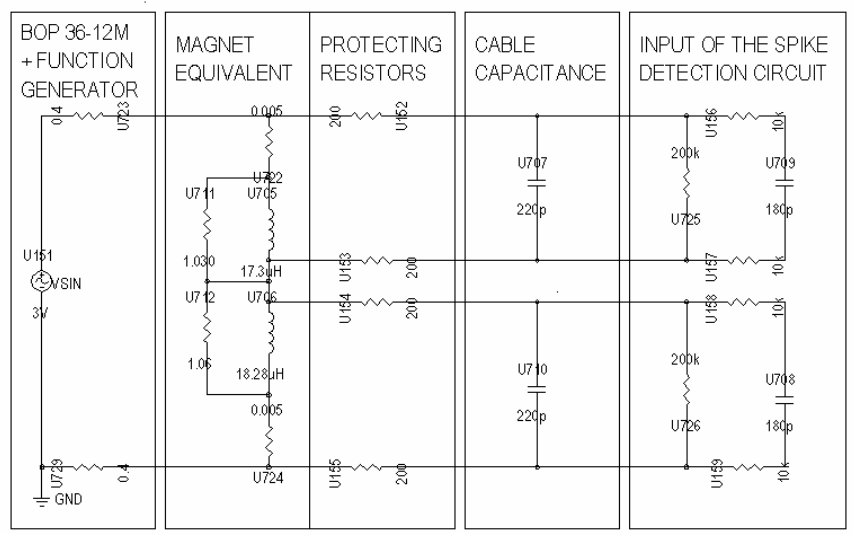

Fig. 2. Circuit used for modeling of the magnet electrical parameters.

A Spice based PCAD Mixed-Signal Simulator was used to obtain the simulation results. Comparison shows that the simulation results agree quite well with the measurement data. This means that the electrical characteristics of the magnet can be modeled with a simple circuit consisting of two inductors and four resistors. The results of this simulation can be seen in Fig. 3.

In the graph the boxes curve represents the measurement data and the triangles curve represents the simulation results. The measurement data was obtained by applying voltage to the magnet via a series resistor - limiting current for low frequencies - and then subtracting the half coil voltages. 


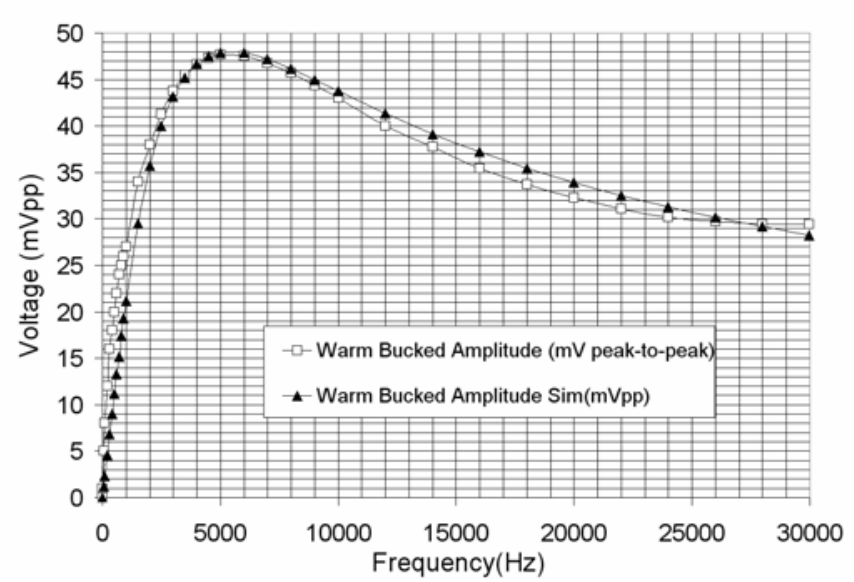

Fig. 3. Simulation and Measurement Comparison of the Superconducting Magnet Half Coil Voltages along with the Bucked Coils Signal as a Function of Frequency.

Adjusting the corresponding components of the model will affect the shape of different sections of the simulation curve. The lower frequency section is determined by the magnitude of the series resistors. Adjusting the difference in the inductance will modify the middle frequency range around the peek value. Introducing an imbalance of the resistance, which represents the magnet losses parallel to the inductors, will modify the high frequency region. Although this model neglects mutual inductance between inductors it produces good results.

\section{System Tests and Verification}

To verify the system performance the following tests have been conducted:

1) Common mode voltage rejection bench test.

2) Common mode voltage rejection in-situ test.

3) Bucking error test and frequency response verification for each channel of the spike detection system.

The common mode voltage rejection bench test circuit

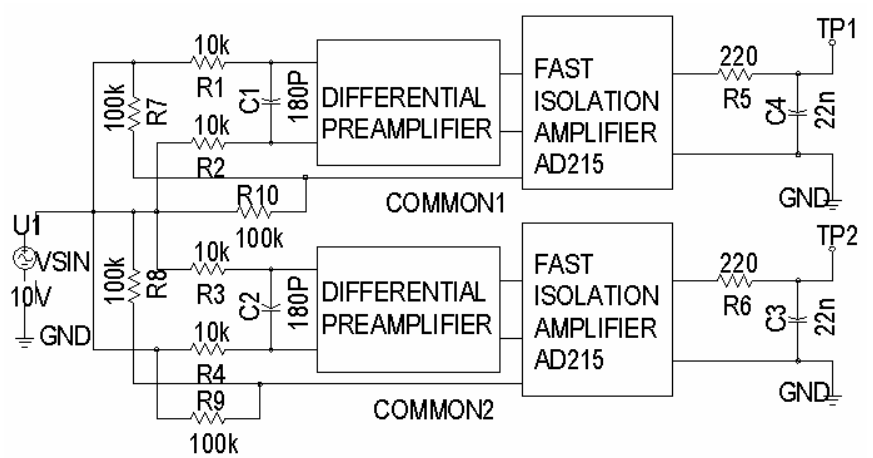

Fig. 4. Schematic of the bench test circuit for the voltage common mode rejection measurement of the spike detection system.

configuration is shown in Fig. 4.

Shorted inputs of both channels were connected to the 20Vpp output of a function generator. Output voltage amplitudes on each channel of isolation amplifier were then recorded as well as the bucking signal. The test was performed for many frequencies to cover the full design range of the spike detection system: 0 to $30 \mathrm{kHz}$. The gain of the differential preamplifiers was typically set to 21 . It was found that the amplitudes of all measured signals were significantly below $1 \mathrm{mV}$, confirming that the voltage common mode rejection ratio is greater than $106 \mathrm{~dB}$.

For the in-situ common mode voltage rejection test the shorted inputs of the spike detection system were connected as follows: One input to the center tap of the magnet and the second input to the positive power supply terminal tap of the magnet. Similar to the bench test, the results obtained during the normal operation of the power supply confirmed that minimal common mode voltage was measured on the input channels and the bucked signal as well.

To perform the bucking error test the two inputs of the spike detection system were connected in parallel and then attached to a function generator set to $1 \mathrm{Vpp}$. While sweeping through the design frequency range the function generator amplitude and bucked signal voltage was recorded for many frequencies. This test was also conducted for excitations lower than $1 \mathrm{Vpp}$. The bucked signal voltage was measured $<1 \mathrm{mVpp}$ for all input voltages and frequencies.

\section{E. Spike Signal Triggering}

In order to capture spike events, the data acquisition system must be able to trigger on real spikes, not on noise. Though both channels are well balanced there are still small DC offsets of the amplifiers, which degrades the ability to digitally buck. This problem is exasperated if the inductances of the magnet half coils are not exactly equal. It can be seen in Fig. 5 that the SNR of the digitally bucked raw half coil signal is not good enough to be able to trigger confidently on a real

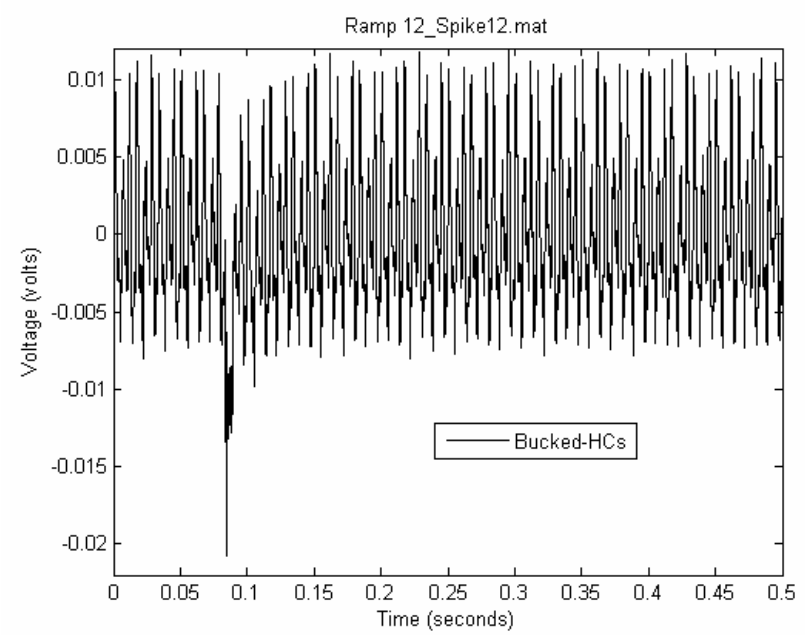

Fig. 5. Bucked, raw, superconducting half coils at 4950A and 20A/s ramp rate. The data was sampled at $100 \mathrm{KHz}$.

magnet spike signal.

In addition to a small DC offsets there is also $720 \mathrm{~Hz}$ noise present in the power supply current, and other harmonics, which shows up in the magnet as voltage noise. To overcome this problem a linear phase digital bandpass filter was implemented in real time. This filter was designed to reject signals below $1500 \mathrm{~Hz}$, including DC, and all signals beyond the bandwidth of interest, $>30 \mathrm{KHz}$. Due to the high sample rate of the ADC, such a filter required 800 taps. Fig. 6 shows 
both the magnitude and phase response of the finite impulse response (FIR) filter designed for use with the spike
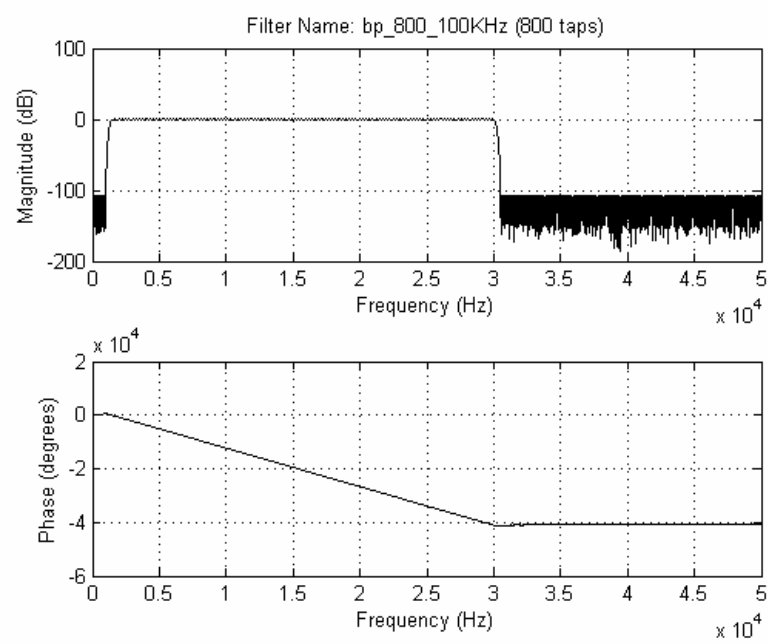

Fig. 6. Magnitude and Phase Response of the digital bandpass FIR filter.

measurement trigger.

The data acquisition algorithm transfers data from First-In First-Out (FIFO) memory in blocks, which are then filtered and bucked. Since the data is block filtered the first $\mathrm{N}$ samples - where $\mathrm{N}$ is the number of filter taps - are ignored due to zero padding of the filtering algorithm. Zero padding at the beginning of the data block can produce artificial transients, which could cause false triggers. Ignoring these first samples results in a dead time; however, this dead time is very small: $800 \mathrm{taps} / 100 \mathrm{KHz}=0.008 \mathrm{~s}$. Since each block of data is $0.5 \mathrm{~s}$ in length then the percent dead time $(\% \mathrm{DT})$ is $\% \mathrm{DT}=$ $(0.008 / 0.5) * 100=1.6 \%$.

This algorithm worked very well and the system has no trouble keeping up with the continuous filtering of the two half coil signals using the 800tap filter at the $100 \mathrm{kHz}$ sample

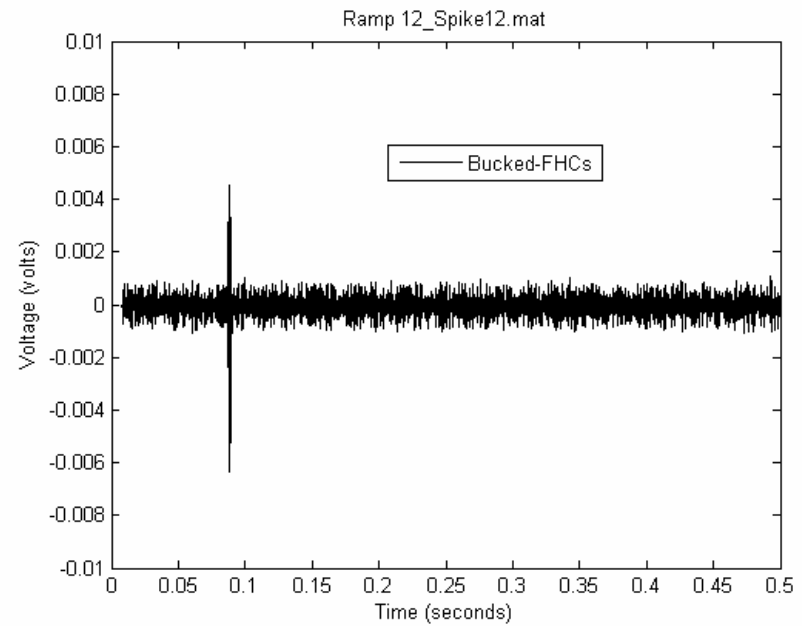

Fig. 7. Bucked, digitally filtered, superconducting half coils at 4950A and $20 \mathrm{~A} / \mathrm{s}$ ramp rate. The data was sampled at $100 \mathrm{KHz}$.

rate. Fig. 7 shows the improved results of the bucked filtered half coils. The SNR is clearly much better.

Since the magnet inductance varies for different magnets the magnitude of the noise is different for different magnets.
In addition, the power system's analog noise filters are dependent on current and therefore the SNR of the resultant signal varies as the magnet current is ramped. For this reason, the threshold setting must be dynamic, and although the operator can change this setting manually it is more dependable to use an automatic trigger level algorithm. A simple method that was employed, measured the standard deviation of the signal where no spike appeared, and then sets the trigger threshold at a level equal to ten standard deviations of the bucked filtered half coil signal.

\section{FINAL RESUlTS}

\section{A. Testing High Filed Magnets}

The spike detection system has been completed and successfully tested on a high-field Nb3Sn superconducting magnet. Data for more than 100 triggered spike events has been collected and analyzed.

Since greater emphasis in the High Field Magnet program has recently been placed on instability studies, feedback from spike studies should prove to be a valuable tool for the development of high field superconducting magnets [4].

\section{B. Spike Signal Analysis}

The data collected from the spike detection system is written to both ASCII files as well as Matlab compatible files, since analysis is performed in the Matlab environment.

A graphical user interface (GUI) has been developed via Matlab that allows users to read and analyze data. It has been compiled as a stand-alone executable so it can be run from any PC. It includes navigation tools, DSP filtering, bucking of coil signals, differentiation, and visualization tools.

\section{CONCLUSIONS}

A superconducting voltage transient "spike" detection system has been designed, built, and tested. Simulations and special tests were carried out in order to characterize the performance of the system, which aided in the engineering and specification of the final product. The real-time Labview DAQ software provides the user with a detailed yet practical user interface that performs auto-triggering, and diagnostics capabilities via pseudo real-time FFT tools. The system also includes a practical graphical user interface with tools for data analysis. Tests on high field magnets are in progress.

\section{REFERENCES}

[1] D. F. Orris, "A Digital Quench Detection System for Superconducting Magnets", Published in Proc. Particle Accelerator Conference, 1999, New York, vol. 5 pp. 3191-3193.

[2] A. F. Lietzke, LBNL, Berleley, CA, private communication, April 2004.

[3] R. Carcagno, "Ripple Current Effects on the Performance of Superconducting High Filed Magnets using a New 30KA Power System at Fermilab," IEEE Trans. Enabling Technologies, submitted for publication.

[4] S. Feher, "Sudden Flux Change Studies in High Field Superconducting Accelerator Magnets", IEEE Trans. Enabling Technologies, submitted for publication 